\title{
APLICAÇÃO DA ENERGIA DE MICRO-ONDAS PARA SECAGEM DA BANANA NANICA (Musa acuminata 'Dwarf Cavendish') SUBMETIDA AO PROCESSO DE OSMOSE
}

\section{APPLICATION OF MICROWAVE ENERGY FOR DRYING BANANA NANICA (Musa acuminata 'Dwarf Cavendish') SUBMITTED TO THE OSMOSIS PROCESS}

Lara Beatriz Geromel, Irene Rodrigues Freitas

Centro Universitário do Norte Paulista - UNORP, Curso de Engenharia Química, SP. E-mail: blarageromel@gmail.com; irfreitas@yahoo.com.br

RESUMO - No Brasil a banana é extremamente importante, pois é a segunda fruta mais cultivada depois das cítricas. Em vista disso, este trabalho teve como objetivo efetuar a desidratação osmótica da banana nanica (Musa acuminata 'Dwarf Cavendish') associada à secagem por micro-ondas. Foi realizada a caracterização da matériaprima, a desidratação e a secagem em micro-ondas doméstico, a cinética de secagem, capacidade de reidratação e caracterização do produto final. Como resultado notou-se que a secagem por microondas foi satisfatória para secagem. Foi obtido um teor de umidade de $29,38 \%$, um pH de 4,77 e uma capacidade de reidratação de $174 \%$ na banana sem o pré-tratamento e $32,33 \%$, 4,83\% e $120 \%$ respectivamente, para a desidratada osmoticamente. 0 uso dessa combinação mostrou-se uma alternativa viável no prolongamento da vida útil da banana nanica.

Palavras-chave: caracterização; desidratação; tecnologia não convencional.

ABSTRACT - In Brazil, bananas are extremely important because they are the second most cultivated fruit after citrus. Therefore, this work aimed to perform the osmotic dehydration of the dwarf banana (Musa acuminata 'Dwarf Cavendish') associated with microwave drying. The characterization of the raw material, dehydration and domestic microwave drying, drying kinetics, rehydration capacity and characterization of the final product were performed. As a result it was noted that microwave drying was satisfactory for drying. A moisture content of $29.38 \%$ was obtained, a pH of 4.77 and a rehydration capacity of $174 \%$ in banana without pretreatment and $32.33 \%, 4.83 \%$ and $120 \%$ respectively for osmotically dehydrated. The use of this combination proved to be a viable alternative in extending the life of the Dwarf Cavendish.

Keywords: characterization; dehydration; unconventional technology. 


\section{INTRODUÇÃO}

A banana é uma fruta de elevado valor nutricional. É boa fonte energética, possuindo alto teor de carboidratos - amido e açúcares. Contém ainda teores consideráveis de vitaminas $A, B 1$ (tiamina), $\mathrm{B} 2$ (riboflavina) e $\mathrm{C}$ e de sais minerais como potássio, fósforo, cálcio, sódio e magnésio, além de outros em menor quantidade (EMBRAPA, 2004).

Sendo a segunda fruta mais cultivada no Brasil, o cultivo é popular em todas as regiões, mas ainda enfrenta perdas expressivas (ARAÚJO, 2016), cerca de $40 \%$ da produção total do país (SEBRAE NACIONAL, 2016).

Segundo dados do IBGE a safra nacional de 2017, referente a janeiro deste mesmo ano, contou com $477.261 \mathrm{mil}$ hectares de plantação e 6.778 .043 milhões de toneladas de bananas produzidas (IBGE, 2017). A safra brasileira da banana é a quarta do mundo, mas o país já está no topo do pódio do consumo global. A fruta rendeu $R \$$ 14 bilhões, no ano de 2016 (GOMES, 2017).

Devido a essa versatilidade e alto consumo pela população, a banana possui ótima relação de custo-benefício. "É uma cultura fantástica, principalmente para a agricultura familiar. Os cuidados são simples e é uma fruta muito consumida, o que oferece estabilidade ao pequeno produtor", destaca. A variedade mais consumida é a banana nanica, seguida pela maçã e prata (STRINI, 2017).

O primeiro levantamento da safra de banana para 2018/19 indicou um aumento de $3,9 \%$ na produção, em relação à safra 2017/18, projetando 1,11 milhão de toneladas da fruta. A expectativa é que a área em produção alcance 58,7 mil ha e aumento de $1,0 \%$ em relação à safra anterior, e a banana é a segunda fruta mais produzida no Estado de São Paulo, perdendo apenas para a laranja, no ranking das frutas consumidas in natura (FREDO et al., 2019). A banana nanica, pertence ao grupo genômico AAA e subgrupo Cavendish de Musa acuminata Colla. Também podendo ser referida como Dwarf Cavendish, e é uma variedade facilmente encontrada nos mercados. Porém é uma fruta susceptível a grandes perdas, com apenas $60 \%$ da produção aproveitada, devido a composição química. Para tentar diminuir parte da perda da banana, foram analisados alguns métodos de preservação de alimentos e dentre eles está à desidratação osmótica.

A desidratação osmótica é uma técnica que pode ser empregada preliminarmente à secagem convectiva e visa à melhoria da qualidade da fruta seca, uma vez que minimiza os danos causados à textura e ao sabor do alimento pelo calor, além de permitir a obtenção de um produto mais atraente para o consumidor (EMBRAPA, 2016).

Essa desidratação consiste na imersão do alimento sólido, inteiro ou em pedaços, em soluções aquosas concentradas de açúcares ou sais, levando a dois fluxos de massa simultâneos: fluxo de água do alimento para a solução devido à diferença na pressão osmótica e transferência simultânea de soluto da solução para o alimento, devido aos gradientes de concentração (TORREGGIANI, 1993).

Como um tratamento para secagem prévia de alimentos sólidos, a desidratação osmótica reduz a quantidade de água presente nos alimentos causando uma melhora nas características sensoriais e físico-químicas do produto, e como resultado o prolongamento da vida útil dos alimentos. A desidratação e / ou secagem geram um alimento de menor consistência e menor tamanho, de bom sabor e odor, muito resistente e de fácil transporte (GARCÍAPATERNINA et al., 2015)

O tratamento é usado principalmente em alguns processos convencionais, tais como secagem a ar convectivo, micro-ondas e liofilização (SERENO et al., 2001).

A secagem com aquecimento em micro-ondas tem como vantagem o controle do aquecimento e a eficiência do uso da energia (SCHIFFMANN, 2001). A radiação micro-onda está dentro do espectro 
eletromagnético no intervalo de frequências entre $300 \mathrm{MHz}$ e $300 \mathrm{GHz}$, com comprimentos de onda variando $1 \mathrm{~mm}$ a $1 \mathrm{~m}$ respectivo a frequência (PEREIRA, 2007).

Os fornos de micro-ondas e quando utilizados reduzem significativamente os ciclos de secagem (SKOOG et al., 2006). Este equipamento usa ondas eletromagnéticas de alta frequência, na faixa de $2450 \mathrm{MHz}$, frequência essa igual à das moléculas da água, motivo pelo qual a energia é diretamente transferida às moléculas de água dos alimentos, possibilitando o seu cozimento (FRANCO, 2018).

Diante do exposto, e devido ao aumento da demanda por alimentos prontos para consumo, aplicar tecnologias não convencionais, como a energia de microondas para secagem associada com uma etapa de pré-tratamento é necessário para melhorar o processo quanto à qualidade de frutas. Este trabalho teve como objetivo efetuar a desidratação osmótica da banana nanica (Musa acuminata 'Dwarf Cavendish') e avaliar a eficiência da cinética de secagem em função da desidratação osmótica associada á secagem por micro-ondas.

\section{MATERIAL E MÉTODOS}

\subsection{MATÉRIA - PRIMA}

As análises para obtenção das variáveis foram realizadas no Laboratório de Química do Centro Universitário do Norte Paulista (UNORP). No experimento foram utilizadas bananas nanicas da produção regional e adquiridas no Mercado do Norfo, localizado em Palmares Paulista - SP (Latitude: -21.0805, Longitude: $-48.804221^{\circ}$ 4' 50" Sul, 48 48' 15" Oeste).

As pencas pré-selecionadas encontravam-se no estágio de maturação, maduras, apresentando-se amarelas e com algumas manchas pretas presentes na casca.

\subsection{MÉTODOS}

\subsubsection{CARACTERIZAÇÃO DA MATÉRIA-PRIMA}

A banana nanica foi caracterizada quanto:
- $\mathrm{pH}$ - Obtido por leitura direta em pHmetro (marca Quimis; modelo 400A). Foi realizado o preparo de uma solução com amostras de bananas, e posteriormente realizadas as medições de $\mathrm{pH}$, segundo o método AOAC (2000).

- Teor de umidade - Realizada por secagem direta em estufa a $105^{\circ} \mathrm{C}$.

- As amostras com peso de 2 a $10 \mathrm{~g}$ foram acondicionadas em placas de Petri e pesadas em balança analítica com quatro casas decimais; a função tara foi utilizada de forma a subtrair o peso da placa de Petri. Em seguida o material (banana) foi aquecida durante o intervalo de uma hora e posteriormente resfriada em dessecador até atingir a temperatura ambiente; logo após foram pesadas, conforme o método da AOAC (2000). O resultado foi obtido através equação (1).

$$
\text { Umidade }=\frac{100 * \mathrm{~N}}{\mathrm{P}}
$$

Onde:

$\mathrm{N}=\mathrm{n}^{\circ}$ de gramas de umidade (perda de massa em $g$ )

$$
\mathrm{P}=\mathrm{n}^{\circ} \text { de gramas da amostra }
$$

- Densidade aparente realizada por ensaio em proveta. As bananas foram fatiadas em frações homogêneas e inseridas em proveta de vidro com capacidade de $50 \mathrm{~mL}^{-1}$; a massa da proveta vazia foi previamente pesada e após a inserção da banana colocada até a marca da graduação foi novamente pesada. O resultado foi obtido utilizando a seguinte equação.

$$
\rho_{\text {aparente }}=\frac{m_{\text {amostras }}}{V_{\text {total }}}
$$

Onde:

$$
\begin{aligned}
& \rho_{\text {aparente }}=\text { densidade aparente }\left(\mathrm{gmL}^{-1}\right) \\
& \mathrm{m}_{\text {amostra }}=\text { massa da amostra }\left(\mathrm{g}^{-1}\right) \\
& \mathrm{V}_{\text {total }}=\text { Volume total }(\mathrm{mL})
\end{aligned}
$$


2.2.2 DESIDRATAÇÃO OSMÓTICA DA BANANA

Foram preparadas três soluções saturadas de água e açúcar (marca Caravelas $^{\circledR}$ ) com proporções de 50/50, 60/40 e 65/35 de açúcar/água; pesada em balança analítica. Para definir qual a melhor condição foram utilizadas diversas concentrações conforme o planejamento experimental do tipo $2^{3}$ apresentado na Tabela 1 . As variáveis reais foram escolhidas depois de uma pesquisa com outros autores para referência de parâmetros.

Tabela 1. Matriz do planejamento experimental fatorial completo do tipo $2^{3}$ utilizada para obtenção da banana osmoticamente desidratada.

\begin{tabular}{ccccccc}
\hline Ensaios & \multicolumn{3}{c}{ Variáveis codificadas } & \multicolumn{3}{c}{ Variáveis reais } \\
\cline { 2 - 7 } & $\mathbf{X 1}$ & $\mathbf{X 2}$ & $\mathbf{X 3}$ & $\begin{array}{c}\text { Tempo de } \\
\text { Imersão } \\
(\mathbf{m i n})\end{array}$ & $\begin{array}{c}\text { Concentração } \\
\text { (g) }\end{array}$ & $\begin{array}{c}\text { Temperatura } \\
\left.\text { ( }{ }^{\circ} \mathbf{C}\right)\end{array}$ \\
\hline 1 & -1 & -1 & -1 & 15 & 50 & 30 \\
2 & +1 & -1 & -1 & 60 & 50 & 30 \\
3 & -1 & +1 & -1 & 15 & 65 & 30 \\
4 & +1 & +1 & -1 & 60 & 65 & 30 \\
5 & -1 & -1 & +1 & 15 & 50 & 65 \\
6 & +1 & -1 & +1 & 60 & 50 & 65 \\
7 & -1 & +1 & +1 & 15 & 65 & 65 \\
8 & +1 & +1 & +1 & 60 & 65 & 65 \\
9 & 0 & 0 & 0 & 30 & 60 & 45 \\
10 & 0 & 0 & 0 & 30 & 60 & 45 \\
11 & 0 & 0 & 0 & 30 & 60 & 45 \\
\hline
\end{tabular}

X1= Tempo de imersão; X2= Concentração; X3= Temperatura.

Fonte: Os autores.

As bananas in natura fatiadas em rodelas de aproximadamente $1 \mathrm{~cm}$ foram submergidas nas soluções devidamente homogeneizadas

O experimento foi realizado em triplicata para cada ensaio.

\subsubsection{SÓLIDOS SOLÚVEIS TOTAIS (SST)}

Para determinar o teor de sólidos solúveis totais, uma parte de fruta foi diluída e agitada na proporção de três partes de água e uma de fruta (em massa) durante 1 minuto. Com auxílio de uma espátula parte da solução, foi cuidadosamente posicionada no prisma do refratômetro portátil (marca Akso; modelo RAB-32 ATC) para leitura direta do teor de sólidos solúveis totais em ${ }^{\circ} \mathrm{Brix}$. $\mathrm{O}$ SST foi realizado na banana in natura, nas condições de desidratação.

\subsubsection{SECAGEM DAS BANANAS DESIDRATADAS OSMOTICAMENTE EM MICRO-ONDAS}

A secagem foi efetuada em um microondas doméstico (Consul, modelo: CMS18), com potência máxima de $800 \mathrm{~W}$. As fatias foram postas sobre um suporte furado de polipropileno (Figura 1), para maior contato com a energia micro-ondas. A cada 1 minuto de secagem, utilizando a potência máxima, as bananas foram pesadas para a construção da curva de cinética de secagem. 
Figura 1. Suporte de polipropileno e micro-ondas utilizado para a secagem.

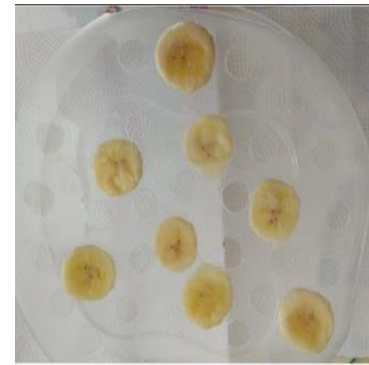

(a)

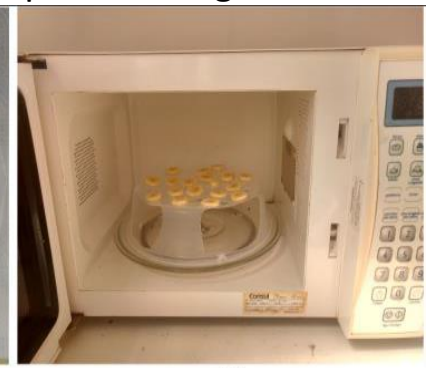

(b) (a) com fatias de banana (b) dentro do micro-ondas, iniciando secagem.

Fonte: Os autores.

\subsubsection{CINÉTICA DE SECAGEM}

A cinética de secagem deu-se pela perda de umidade em função do tempo de secagem das fatias de banana. A equação (1) foi utilizada para calcular a perda de massa da fruta com desidratação osmótica (CDO) e sem desidratação (SDO).

\subsubsection{CARACTERIZAÇÃO DO PRODUTO FINAL}

O produto após a secagem utilizando a energia de micro-ondas foi submetido a análises de $\mathrm{pH}$ e teor de umidade, cujas metodologias estão supracitadas abaixo. As amostras de banana inda foram analisadas quanto a:

\section{- Capacidade de reidratação}

A quantia de $150 \mathrm{~mL}$ de água destilada inserida em um béquer de $500 \mathrm{~mL}$ e mantida em ebulição por 3 minutos. Após esse período, aproximadamente $5 \mathrm{~g}$ da amostra seca foram adicionados à água em ebulição e mantidos por 5 minutos. Após esse período de tempo a amostra reidratada e posteriormente transferida para uma peneira para retirar o excesso de água. Depois de retirado o excesso, as amostras foram pesadas e a capacidade de reidratação calculada conforme a Equação 3, (RIBEIRO, 2013).

$$
C R(\%)=\left(\frac{W_{\text {rei }}-W_{\text {sec } a}}{W_{\text {sec } a}}\right) * 100
$$

Onde:

$\mathrm{CR}(\%)=$ Coeficiente de reidratação;

$\mathrm{W}_{\text {rei }}=$ Massa da amostra reidratada (g);

$\mathrm{W}_{\text {seca }}=$ Massa da amostra desidratada (g).

\subsubsection{ANÁLISE ESTATÍSTICA}

Para averiguação dos efeitos principais e das interações entre fatores foi realizada análise de variância (ANOVA), e a diferença entre os resultados obtidos foi realizado o teste de Tukey. As diferenças foram consideradas significativas quando $p \leq$ 0,05 utilizando o software Statistica ${ }^{\circledR}$ 7.0.

\section{RESULTADOS E DISCUSSÃO}

\subsection{CARACTERIZAÇÃO DA MATÉRIA-PRIMA}

$\mathrm{Na}$ Tabela 3 estão apresentados os dados obtidos a partir da banana in natura. Os valores de $\mathrm{pH}$ e ${ }^{\circ}$ Brix se mostram próximos aos encontrados por Hattenhauer et al. (2016), em que obtiveram valores de 4,64 e 19,56, respectivamente.

Tabela 3. Caracterização da matéria-prima.

\begin{tabular}{cc}
\hline Análises & Resultados \\
\hline $\mathrm{pH}$ & $4,67 \pm 0,04$ \\
SST ( ${ }^{\circ}$ Brix $)$ & $19,30 \pm 0,80$ \\
Umidade $(\%)$ & $56,89 \pm 1,39$ \\
Densidade & $0,60 \pm 0,21$ \\
aparente $(\mathrm{g} / \mathrm{mL})$ & \\
\hline Os autores. &
\end{tabular}

A umidade e a densidade aparente obtiveram valores abaixo ao de Pereira et al. (2007) que obteve umidade de 74,54 g/100g para banana nanica, essa diferença pode ser atribuída ao modo de cultivo, período da colheita e região de plantação, além dos parâmetros de secagem utilizados. Monteiro et al. (2016) encontraram para densidade aparente um valor médio de $1,040 \mathrm{~g} / \mathrm{mL}$ para banana prata, valor superior ao verificado pelo presente estudo, esse fato pode estar relacionado a cultivar avaliada, que podem apresentar certas diferenças.

\subsection{DESIDRATAÇÃO OSMÓTICA}

Na Tabela 4 estão apresentados os resultados para os ensaios da matriz do planejamento experimental. Apesar do SST no ensaio 8 obter o maior valor, que pode ser explicado devido a ser o ensaio com valores de variável codificada positivo ou valores de variável real de maior valor, porém nenhum dos 
resultados diferiram estatisticamente entre si $(p \leq 0,05)$.

Tabela 4. Análises de Sólidos Solúveis Totais ( ${ }^{\circ}$ Brix), pH para banana desidratada osmoticamente.

\begin{tabular}{ccc}
\hline Ensaios & SST & pH \\
\hline 1 & $24,33 \pm 0,94^{\mathrm{a}}$ & $4,75 \pm 0,02^{\mathrm{a}}$ \\
2 & $23,43 \pm 0,42^{\mathrm{a}}$ & $4,66 \pm 0,01^{\mathrm{a}}$ \\
3 & $27,50 \pm 0,27^{\mathrm{a}}$ & $4,73 \pm 0,02^{\mathrm{a}}$ \\
4 & $26,33 \pm 0,47^{\mathrm{a}}$ & $4,69 \pm 0,03^{\mathrm{a}}$ \\
5 & $24,50 \pm 0,41^{\mathrm{a}}$ & $4,72 \pm 0,01^{\mathrm{a}}$ \\
6 & $26,67 \pm 0,47^{\mathrm{a}}$ & $4,65 \pm 0,01^{\mathrm{a}}$ \\
7 & $25,50 \pm 0,41^{\mathrm{a}}$ & $4,55 \pm 0,03^{\mathrm{a}}$ \\
8 & $37,83 \pm 0,24^{\mathrm{a}}$ & $4,52 \pm 0,10^{\mathrm{a}}$ \\
9 & $25,17 \pm 0,24^{\mathrm{a}}$ & $4,54 \pm 0,15^{\mathrm{a}}$ \\
10 & $25,33 \pm 0,47^{\mathrm{a}}$ & $4,52 \pm 0,14^{\mathrm{a}}$ \\
11 & $25,67 \pm 0,47^{\mathrm{a}}$ & $4,50 \pm 0,15^{\mathrm{a}}$ \\
\hline
\end{tabular}

Letras iguais na mesma coluna não diferem entre si pelo teste de Tukey $(p \leq 0,05)$.

Fonte: Os autores.

Segundo Pereira (2007), o valor encontrado para o SST em um ensaio nas condições de solução de sacarose a $55^{\circ}$ Brix, $40^{\circ} \mathrm{C}$ durante 90 minutos foi de $23,2^{\circ}$ Brix, sendo essas condições aproximadas com o experimento 2 que obteve 23,43 de sólidos solúveis.

Para a análise de $\mathrm{pH}$, observa-se também que não houve variação estatística significativa, podendo concluir que não é relevante ao processo.

Em relação ao efeito das variáveis tempo, temperatura e concentração sobre o $\mathrm{pH}$ e sólidos solúveis totais, verificou-se que o $\mathrm{pH}$ aponta para um efeito negativo de todas as variáveis com influência significativa $(p \leq 0,05)$ para todas os fatores, sendo a temperatura que teve o maior efeito.

A combinação do tempo de imersão e da concentração resultou no menor efeito para o experimento, em contra partida a combinação de concentração de açúcar e tempo obteve o maior efeito sobre o $\mathrm{pH}$.

Para o SST o efeito se manteve com influência positiva para todas as variáveis, porém não foi significativo ( $p \leq 0,05$ ) em nenhuma condição. Atingindo um maior efeito para a concentração de açúcar. Sendo a condição do tempo de imersão combinado com a temperatura o de maior efeito, ao passo que a concentração combinada com a temperatura logrou o menor efeito, conforme pode ser observado na Tabela 5.

Tabela 5. Efeitos estimados para pH e SST.

\begin{tabular}{lcrrr}
\hline Variável & pH & \multicolumn{2}{c}{ SST } \\
\hline & Efeito & Valor P & \multicolumn{1}{c}{ Efeito } & Valor P \\
\hline Média da Interação & 4,634361 & 0,006681 & 26,99057 & 0,085300 \\
Tempo de Imersão (1) & $-0,009679$ & 0,013394 & 3,14805 & 0,170998 \\
Conc. de Açúcar (2) & $-0,024660$ & 0,013393 & 4,59806 & 0,170990 \\
Temperatura (3) & $-0,049660$ & 0,013393 & 3,26806 & 0,170990 \\
1 (L) $\times 2$ (L) & $-0,024402$ & 0,013423 & 2,43273 & 0,171371 \\
1 (L) X 3 (L) & $-0,039402$ & 0,013423 & 4,10270 & 0,171371 \\
\hline $2(L) \times 3(L)$ & $-0,124420$ & 0,013422 & 1,48272 & 0,171364 \\
\hline
\end{tabular}

Fonte: Os autores.

Devido ao efeito não haver uma influência significativa $(p \leq 0,05)$ nos sólidos solúveis totais, os efeitos das variáveis nas condições não foram um fator relevante para a escolha da condição ótima. Sendo assim a escolha foi feita com base na aparência visual da banana após a desidratação e na economia de recursos de materiais.

As fatias desidratadas de $45^{\circ} \mathrm{C}$ e $65^{\circ} \mathrm{C}$ não foram consideradas devido à aparência "derretida" e fibrosa, como demostrado na Figuras 2 (b) e (c), sendo assim, foram 
escolhidas as condições mínimas do planejamento experimental, a temperatura de $30^{\circ} \mathrm{C}$, concentração $\left(50^{\circ} \mathrm{Brix}\right.$ ) e tempo (15 minutos) (Figura a).

Figura 2. Fatias de banana desidratada osmoticamente a diferentes temperaturas.

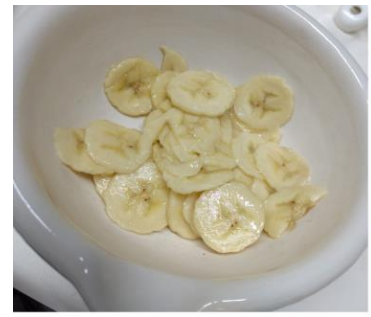

(a)

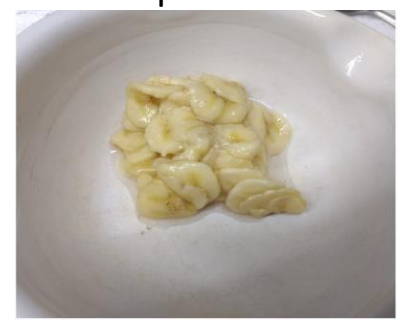

(b)

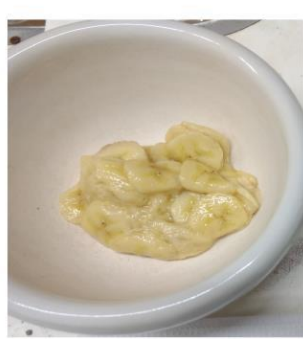

(c)

Temperatura de: (a) $30^{\circ} \mathrm{C}$ (b) $45^{\circ} \mathrm{C}$ (c) $65^{\circ} \mathrm{C}$. Fonte: Os autores.

\subsection{CINÉTICA DE SECAGEM}

A condição da secagem foi referente ao ensaio 1 , onde a temperatura foi de $30{ }^{\circ} \mathrm{C}$ concentração de $50 \mathrm{~g}$ e o tempo de 15 minutos. Na Figura 3 observa-se a cinética de secagem, que foi realizada em 12 minutos.

Figura 3. Cinética de secagem da banana sem desidratação osmótica (SDO) e desidratada osmoticamente (CDO).

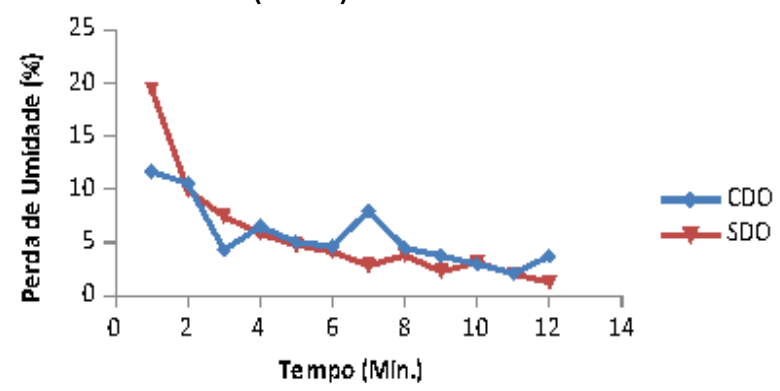

Fonte: Os autores

A partir dos 10 minutos algumas fatias sem desidratação osmótica (SDO) começaram a apresentar leves queimaduras, chegando aos 12 minutos totalmente queimadas (torradas). Enquanto as fatias submetidas à desidratação osmótica (CDO) demoraram até os doze minutos para começarem a apresentar queimaduras.

A maior perda de umidade ocorreu no primeiro minuto, sendo aproximadamente $19,40 \%$ (SDO) e $11,66 \%$ (CDO) e decaindo até uma perda de $1,28 \%$ e $3,78 \%$, respectivamente, no final dos 12 minutos. Observa-se o efeito (Figura 3) da desidratação osmótica na curva formada, tendo em comparação com a banana sem desidratação, uma linha com quedas cinéticas maiores.

\subsection{CARACTERIZAÇÃO DO PRODUTO FINAL}

Dispostos na Tabela 6 os dados da banana sem desidratação osmótica (SDO) e com desidratação osmótica (CDO) após a secagem no micro-ondas, e na Figura 4 observa-se a aparência final das fatias.

Figura 4. Fatias de bananas secas em microondas.
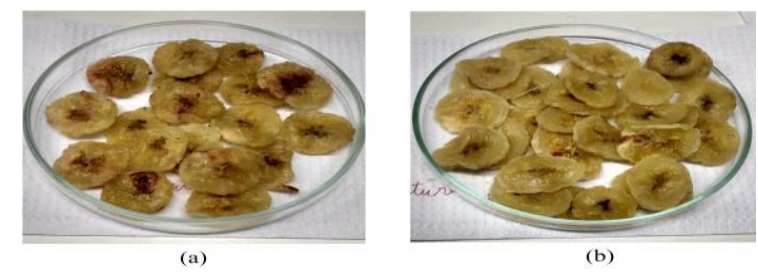

(a) Com desidratação (b) Sem desidratação. Fonte: Os autores.

Observa-se uma baixa variação de $\mathrm{pH}$ entre as bananas sem e com desidratação osmótica. Em geral é desejável pH inferior a 5, para impedir o desenvolvimento de microrganismos patogênicos no produto, logo pode-se inferir que ambas atingiram o pH necessário.

Durante o processo de secagem ocorre a perda de água, e com isso as bananas podem ser conservadas por um período maior e serem transportadas com mais facilidade. Os resultados de umidade deste estudo foram maiores que ao realizado por Rodrigues et al. (2013) que obtiveram $25,54 \%$, para banana desidratada utilizando secagem com circulação de ar que pode explicar a diferença no resultado. 
Tabela 6: Caracterização do produto final.

\begin{tabular}{lcc}
\hline Análises & Banana SDO & Banana CDO \\
\hline $\mathrm{pH}$ & $4,77 \pm 0,03$ & $4,83 \pm 0,01$ \\
Umidade (\%) & 29,38 & 32,33 \\
Capacidade de reidratação (\%) & $174,00 \pm 0,02$ & $120,00 \pm 0,01$ \\
\hline
\end{tabular}

Fonte: Os autores.

A capacidade de reidratação da banana submetida ao processo de osmose foi inferior a não submetida ao pré-tratamento. Como a reidratação é uma consequência da porosidade e do encolhimento causado pelo processo de secagem, a incorporação de sólidos na desidratação osmótica, diminui os espaços vazios dificultando a entrada de água no interior da matriz.

As fatias secas da fruta com e sem desidratação apresentaram uma cor caramelizada. A fruta seca apresentou visualmente um encolhimento se comparado à fruta in natura.

\section{CONSIDERAÇÕES FINAIS}

A desidratação osmótica observada pela ótica de sólidos solúveis totais e de $\mathrm{pH}$ é satisfatório e nas condições referente ao ensaio 1 (Temperatura $30{ }^{\circ} \mathrm{C}$, Concentração $50 \mathrm{~g}$ e Tempo 15 minutos) do planejamento experimental fatorial tipo $2^{3}$, podendo tornarse um ponto positivo se comparado aos outros ensaios devido ao baixo consumo de açúcar, água, energia para o aquecimento e tempo.

A potência de $800 \mathrm{~W}$ para a secagem em micro-ondas é satisfatória, visto que foi possível retirar $67,67 \%$ de água das amostras.

Portanto, a combinação dessas tecnologias pode ser uma alternativa para prolongar a vida útil de frutas, melhorando a qualidade, como a banana.

\section{REFERÊNCIAS}

ASSOCIATION OF OFFICIAL ANALYTICAL CHEMISTS (AOAC). Official Methods of Analysis of the Association of Official Analytical Chemists. Arlington: AOAC, 2000.

ARAÚJO, N. Banana em caixas e melhor climatização podem reduzir as perdas da fruta. Farming Brasil, 2016. Disponível em: https://sfagro.uol.com.br/banana/. Acesso em: 27 maio 2018.

IBGE. Levantamento sistemático da produção agrícola: Pesquisa mensal de previsão e acompanhamento das safras agrícolas no ano civil. Rio de Janeiro: IBGE,2017. Disponível em: ftp://ftp.ibge.gov.br/Producao_Agricola/Leva ntamento_Sistematico_da_Producao_Agricol a_[mensal]/Fasciculo/2017/lspa_201701.pdf. Acesso em: 27 maio 2018.

CORRÊA, J. L. G. et al. Analysis of osmotic dehydration variables influences on tomato (Licopersicon esculentum) drying. Boletim do Centro de Pesquisa e Processamento de Alimentos, v. 25, n. 2, p. 315-328, 2011.

EMBRAPA. Empresa Brasileira de Pesquisa Agropecuária. Aspectos nutricionais, 2004. Disponível em: https://www.agencia.cnptia.embrapa.br/Age ncia40/AG01/arvore/AG01_48_41020068055 .html. Acesso em: 12 maio 2020.

EMBRAPA. Empresa Brasileira de Pesquisa Agropecuária. Mamão Formosa desidratado incorporado de frutooligossacarídeo, 2016. Disponível em: https://ainfo.cnptia.embrapa.br/digital/bitstr eam/item/153436/1/folder-mamaodesidratado-Eliseth-Ronielli.pdf. Acesso em: 27 maio 2018.

FRANCO, D. S. Forno Microondas e seu funcionamento. Juiz de Fora: Instituto de Ciências Exatas - Universidade Federal de Juiz de Fora, 2018. Disponível em: http://www.ufjf.br/fisicaecidadania/conteud o/se-quiser-saber-mais-sobre-o-forno- 
microondas-e-seu-funcionamento/. Acesso em: 27 maio 2018.

FREDO, C. E. et al. Previsões e Estimativas das Safras Agrícolas do Estado de São Paulo, $2^{\circ}$ Levantamento, Ano Agrícola 2018/19 e Levantamento Final, Ano Agrícola 2017/18, Novembro de 2018. Análises e Indicadores do Agronegócio, v. 14, n. 2, 2019.

GARCÍA-PATERNINA, M.; ALVIS-BERMUDEZ, A.; GARCIA-MOGOLLON, C. A. Evaluation of the Osmotic Dehydration Pretreatment and Microwave in the Obtaining of Flakes of Mango (Tommy Atkins). Información tecnológica, v. 26, n. 5, p. 63-70, 2015. https://doi.org/10.4067/S0718-

$\underline{07642015000500009}$

GOMES, M. Produção brasileira de banana atinge $\mathrm{R} \$ 14$ bilhões por ano. Correio Braziliense, 23 outubro 2017. Economia Correio Braziliense. Disponível em: https://www.correiobraziliense.com.br/app/n oticia/economia/2017/10/23/internas_econo mia,635500/producao-brasileira-de-bananaatinge-r-14-bilhoes-por-ano.shtml. Acesso em: 27 maio 2018.

HATTENHAUER S. K.; CARVALHO R. I. N. D. Caracterização física e química da banana 'Nanica' em função da época de colheita e do diâmetro do fruto em Corupá, SC. Agropecuária Catarinense, v. 29, n. 2, p. 8083, 2016.

MONTEIRO R. L.; CARCIOFI B. A. M.; LAURINDO J. B. A microwave multi-flash drying process for producing crispy bananas. Journal of Food Engineering, v. 178, 2016. https://doi.org/10.1016/i.jfoodeng.2015.12.0 $\underline{24}$

PEREIRA, N. R.; MARSAIOLI, A.; AHRNÉ, L. M. Effect of microwave power, air velocity and temperature on the final drying of osmotically dehydrated bananas. Journal of Food Engineering, v. 81, n. 1, p. 79-87, 2007. https://doi.org/10.1016/i.jfoodeng.2006.09.0 $\underline{25}$

PEREIRA, N. R. Estudo da aplicação de microondas na secagem de bananas tratadas osmoticamente. 2007. Tese (Doutorado em Engenharia de Alimentos) Universidade Estadual de Campinas, Campinas, 2007.

RIBEIRO, R. C. Tomates semi desidratados obtidos por secagem micro-ondas convectiva precedida por desidratação osmótica. 2013. Dissertação (Mestrado) Engenharia Agrícola. Universidade Federal de Lavras, Lavras, 2013.

RODRIGUES, F. M.; GUERRA, A. F.; RODRIGUES, L. G. da S. M.; CHÁVEZ, D. W. H. Produção de banana (Musa paradisiaca.) desidratada osmoticamente seguida por secagem com circulação de ar e caracterização físico química. Enciclopédia Biosfera, v.9, n. 16, p. 1750-1758, 2013.

SEBRAE. Serviço Brasileiro de Apoio às Micro e Pequenas Empresas. Conheça o mercado da bananicultura, 2016. Disponível em: http://www.sebrae.com.br/sites/PortalSebra e/artigos/conheca-o-mercado-dabananicultura,187b9e665b182410VgnVCM10 0000b272010aRCRD. Acesso em: 27 maio 2018.

SCHIFFMANN, R. F. Microwave process for the food industry. In: DATTA, A. K.; ANANTHESWARAN, R. C. (ed.). Handbook of microwave technology for food applications, [S.I.]: Marcel Dekker Inc, 2001. p. 299-338

SERENO, A. M.; HUBINGER, M. D.; COMESANÃ, J. F.; CORREA, A. Prediction of water activity of osmotic solutions. Journal of Food Engineering, v. 49, n. 1, p. 103-114, $2001 . \quad$ https://doi.org/10.1016/SO2608774(00)00221-1 
SKOOG, D. A.; WEST, D. M.; HOLLER, F. J.; $\mathrm{CROUCH}, \mathrm{S}$. R. Fundamentos de Química Analítica, Tradução da 8a Edição norteamericana, Editora Thomson, São Paulo-SP, 2006.

STRINI, S.; Banana uma fruta versátil de alto consumo populacional. $100 \%$ CAIPIRA $^{\circledR}$, v. 5 , n. 46, p. 8-13, 2017.

TORREGGIANI, D. Osmotic dehydration in fruit and vegetable processing. Food Research International, v. 26, n. 1, p. 59-68, 1993. 